\title{
Impedance Strongly Inductive Two-Layer Media
}

\author{
PhD Vasiliy K. Balkhanov, Prof. Yuriy B. Bashkuev, PhD Ludmila Kh. Angarkhaeva \\ Institute of Physical Materials Science of the Siberian Branch of the Russian Academy of Sciences, Ulan-Ude \\ City, Russia \\ Email: ballar@yandex.ru
}

\begin{abstract}
This paper derives the analytical formula for impedance two-layer media "dielectricconductor". In the winter on the Northern sea route and the salt lakes of Eurasia for demanding radio paths you can model two-layer media, where the first media (ice) is a dielectric layer and the second (sea, salt water) over a wide frequency range of radio waves is underlying conductive media. Two-layer media under consideration with real physical and geometrical parameters has strongly inductive impedance, which is calculated from a transcendental equation. The article shows that a large contrast between the electrophysical properties of the layers of ice and water allows you to set an approximate analytical formula for impedance, which is within the applicability of the impedance definition practically coincides with the decision of the transcendental equation. It was established that for the two-layer media impedance phase lies within $-45.1^{0}--89.9^{0}$, that is the criterion for heavily inductive surface.
\end{abstract}

Keywords: Dielectric (ice), conductor (water) impedance.

\section{Introduction}

We consider the distribution of electromagnetic wave in free space on the surface along two-layer media "dielectric layer thickness endpoint - unlimited conductive underlying media". Free space will be marked with the index 0 . The first dielectric layer is marked with index 1 , which has the thickness $h$ and describes the relative dielectric permeability $\varepsilon_{1}$ and specific electric resistance $\rho_{1}$. The second media is marked with index 2 , with unlimited depth and describes specific electrical resistance $\rho_{2}$ and dielectric permeability $\varepsilon_{2}$. Two-layer under consideration media is a good model of a strongly inductive surface used for spreading along the radio route electromagnetic wave. Our task is to establish the following analytical expression of impedance $\delta$ for the highly inductive for demanding radio paths:

$$
\delta=\sqrt{-i \varepsilon_{0} \omega \rho_{2}}-i \frac{\omega h}{c}\left(1-\frac{1}{\varepsilon_{1}+i / \varepsilon_{0} \omega \rho_{1}}\right)
$$

Here $\varepsilon_{0}=8.85 \cdot 10^{-12} \mathrm{~F} / \mathrm{m}$ - dielectric constant of the vacuum, $c$ - the speed of light, $\omega$ - circular frequency.

It is always better to deal with analytical expression. Urgency stems from the fact that knowledge of the underlying impedance media enough to determine all characteristics for demanding radio paths and characteristics of electromagnetic field in place of the radio reception.

\section{Wavenumber}

Electromagnetic wave, spreading along the Earth for demanding radio paths, is characterized by a wave number $k$. For multilayer media in each homogeneous layer square wave number $k^{2}$ given the following famous expression [1-3]:

$$
k^{2}=\frac{\omega^{2}}{c^{2}}\left(\varepsilon+\frac{i}{\varepsilon_{0} \omega \rho}\right)
$$

In free space $\varepsilon=1$ and $\rho \rightarrow \infty$, then the square of the wave number of free space $k_{0}{ }^{2}=\frac{\omega^{2}}{c^{2}}$. 
For salt lakes of Eurasia in March-April is the first layer of ice with dielectric permeability $\varepsilon_{1}=3.4$, and thickness of order $h=1 \mathrm{~m}$ [4]. Resistivity of ice on salt water $\rho_{1}=10^{4} \Omega \cdot \mathrm{m}$. In the frequency range up to $20 \mathrm{MHz}$ maximum value $1 / \varepsilon_{0} \omega \rho_{1}=9$. It is the same order with $\varepsilon_{1}=3.4$. So for the first layer, you must believe

$$
k_{1}^{2}=\frac{\omega^{2}}{c^{2}}\left(\varepsilon_{1}+\frac{i}{\varepsilon_{0} \omega \rho_{1}}\right)
$$

The second layer of salt lakes of Eurasia is water several meters deep, for which $\varepsilon_{2}=81$ and $\rho_{2}=1$ $\Omega \cdot \mathrm{m}$. Here in the frequency range $100 \mathrm{kHz}-20 \mathrm{MHz}$ value $1 / \varepsilon_{0} \omega \rho_{2}=1 \cdot 10^{6}-1000$, that is much larger than $\varepsilon_{2}=81$. So for the second layer, you can take $k_{2}^{2}=\frac{i \mu_{0} \omega}{\rho_{2}}$.

Here vacuum magnetic constant $\mu_{0}=1 / \varepsilon_{0} c^{2}$. Skin layer [1-3] in the frequency range $100 \mathrm{kHz}-20$ $\mathrm{MHz}$ for the water layer is given by the following expression:

$$
\sqrt{\frac{2 \rho_{2}}{\mu_{0} \omega}}=1.5-0.15 \mathrm{~m}
$$

This value is significantly less than the depths of the Lake, so the second layer can be considered unlimited and not seen the reflection of electromagnetic waves from the lake bottom.

\section{Transcendental Equation for Impedance}

The impedance for two-layer media largely repeats similar calculations for a homogeneous impedance media in [5]. It is only necessary to explicitly take into account the underlying two-layer media. Omitting these calculations, we find the first transcendental equation to determine the parameter $\lambda$ :

$$
\begin{gathered}
\frac{1-Q_{10}}{1+Q_{10}}=\exp \left(2 i \sqrt{k_{1}^{2}-\lambda^{2}} h\right) \frac{1-Q_{12}}{1+Q_{12}} \\
Q_{10}=\frac{k_{1}^{2}}{k_{0}^{2}} \frac{\sqrt{k_{0}^{2}-\lambda^{2}}}{\sqrt{k_{1}^{2}-\lambda^{2}}}, \quad Q_{12}=\frac{k_{1}^{2}}{k_{2}^{2}} \frac{\sqrt{k_{2}^{2}-\lambda^{2}}}{\sqrt{k_{1}^{2}-\lambda^{2}}}
\end{gathered}
$$

Then connect option $\lambda$ with an impedance $\delta$ :

$$
\lambda=k_{0} \sqrt{1-\delta^{2}}
$$

Equation (3) is transcendental and is solved only numerically, for this program is written "Impedance" [6]. However, two-layer under consideration media is a large contrast between the electrophysical properties of the layers of ice and water, which lets you select a different order of magnitude, and when this becomes possible analytical solution of equation (3).

\section{An Approximate Expression for the Impedance}

By definition, a square impedance module satisfies the inequality $|\delta|^{2}<<1$. This allows the neglected in the transcendental equation (3) value $\delta^{2}$. As a result, equation (3) takes the following form:

$$
\frac{1-Q_{10}}{1+Q_{10}}=W \frac{1-Q_{12}}{1+Q_{12}} \equiv K
$$

Here entered the exhibitor

$$
W=\exp \left(2 i \frac{\omega h}{c} \sqrt{\varepsilon_{1}-1+\frac{i}{\varepsilon_{0} \omega \rho_{1}}}\right)
$$


Values $Q_{10}$ and $Q_{12}$ provide expression (4), with $\delta^{2}$ discarded. Next, using the expression (2) for $k_{1}^{2}$, (6) finally finds the following expression for the impedance:

$$
\delta=\frac{\sqrt{\varepsilon_{1}-1+i / \varepsilon_{0} \omega \rho_{1}}}{\varepsilon_{1}+i / \varepsilon_{0} \omega \rho_{1}} \frac{1-K}{1+K}
$$

The resulting expression $(21)$ can be simplified further. For this purpose it is necessary to take into account that $\lambda \approx k_{0}$. In addition, for conductive underlying media in wide frequency range $k_{2}^{2}>>k_{0}^{2}$ and $k_{2}^{2} \gg \lambda^{2}$. These allow for inequalities imposed values $Q_{10}$ and $Q_{12}$ :

where

$$
Q_{10}=\Lambda \delta<<1, Q_{12}=\Lambda \sqrt{-i \varepsilon_{0} \omega \rho_{2}}<<1
$$

$$
\Lambda=\frac{\varepsilon_{1}+i / \varepsilon_{0} \omega \rho_{1}}{\sqrt{\varepsilon_{1}-1+i / \varepsilon_{0} \omega \rho_{1}}}
$$

Then, from (8) first, we have approximately $1-2 \Lambda \delta=W\left(1-2 \Lambda \sqrt{-i \varepsilon_{0} \omega \rho_{2}}\right)$.

Hence, the impedance:

$$
\delta=W \sqrt{-i \varepsilon_{0} \omega \rho_{2}}+\frac{1-W}{2 \Lambda}
$$

This expression for the impedance is correct in the assumption that $|\delta|<<1$ and $\sqrt{\varepsilon_{0} \omega \rho_{2}}<<1$.

Expression (10) allows for further simplification. Suppose that argument in the exponent (8) is small. In a number of decomposed exponent:

$$
W \approx 1+2 i \frac{\omega h}{c} \sqrt{\varepsilon_{1}-1+\frac{i}{\varepsilon_{0} \omega \rho_{1}}} .
$$

Then from (10) we get finally the following expression for the impedance:

$$
\delta=\sqrt{-i \varepsilon_{0} \omega \rho_{2}}-i \frac{\omega h}{c}\left(1-\frac{1}{\varepsilon_{1}+i / \varepsilon_{0} \omega \rho_{1}}\right)
$$

The ratio of (1) two important conclusions follow. If the first media in a certain frequency range is conductive (e.g., very dirty ice), i.e. executes the inequality

$$
1 / \varepsilon_{0} \omega \rho_{1} \gg \varepsilon_{1}
$$

the expression of $\left(\varepsilon_{1}+1 / \varepsilon_{0} \omega \rho_{1}\right)^{-1}$ compared to 1 can be neglected. And if we mark $\delta_{2}=\sqrt{-i \varepsilon_{0} \omega \rho_{2}}$, then from (1) we get

$$
\delta=\delta_{2}-i \frac{\omega h}{c}
$$

Thus we find the amendment to the impedance in the presence of a thin conductive layer [2.7]. Note also that the impedance $\delta_{2}$ you can get the exact phrase:

$$
\delta_{2}=k_{0} \sqrt{k_{2}^{2}-k_{0}^{2}} / k_{2}^{2} \text {. }
$$

In the other extreme case, when the first layer is filled with a dielectric permeability $\varepsilon_{1}$, i.e. for him in a certain frequency range runs inequality

$$
1 / \varepsilon_{0} \omega \rho_{1}<<\varepsilon_{1}
$$

then from (1) should be

$$
\delta=\delta_{2}-i \frac{\varepsilon_{1}-1}{\varepsilon_{1}} \frac{\omega h}{c}
$$

Thus we find the amendment to the impedance in the presence of a thin dielectric layer. 


\section{Graphical Representation of the Impedance}

An important representation of the Earth's surface is its simulation of multilayer geoelektric media [8]. To calculate the impedance of multilayer media the software package "Impedance" [6], this program allows a numerical method to solve transcendental equation (3). For certainty from the salt lakes of Eurasia we will choose Lake Sulphate, located in the South of the Republic of Buryatia. The results of calculations for the Lake Sulphate, for which there are known $h=1 \mathrm{~m}, \varepsilon_{1}=3.2, \rho_{1}=10^{4} \Omega \cdot \mathrm{m}$ and $\rho_{2}=1.1 \Omega \cdot \mathrm{m}, \varepsilon_{2}=81$, are presented for the impedance module $|\delta|$ in Figure 1 and impedance phase $\phi_{\delta}$ in Figure 2. Recall that $\delta=|\delta| \exp \left(i \phi_{\delta}\right)$. It also illustrates the result computed by the formula (1). Analysis of the figures shows that the impedance module $|\delta|$ the results are the same up to $20 \mathrm{MHz}$. It has been established that the impedance phase $\phi_{\delta}$ lies within $-45.1^{0}--89.9^{0}$. This indicates that the surface is strongly inductive and can serve for the purposes of long - distance radio communication.

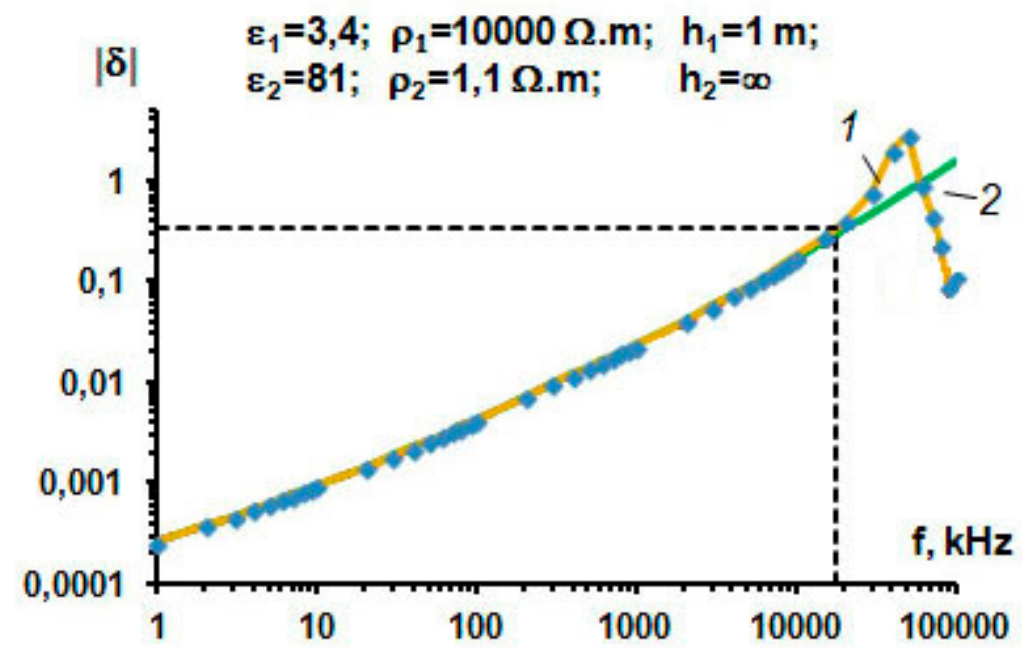

Figure 1. Impedance module dependency of frequency on the structure of "ice on salt water". A solid line with squares 1 is calculated under the "Impedance" [6], the solid line 2 is calculated from equation (1). In a rectangular area bounded by dashed lines, run conditions $|\delta|<<$.

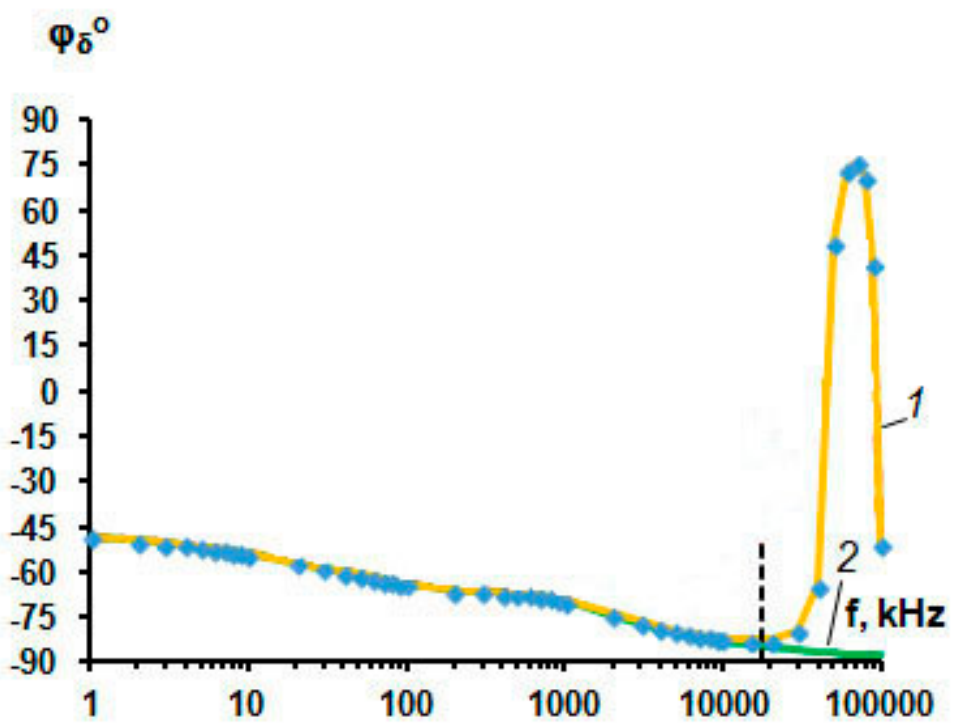

Figure 2. Impedance phase dependence of frequency. (Designations are the same as on the fig. 1.) 
Figures 1 and 2 follow that up to $20 \mathrm{MHz}$ frequency the condition $|\delta|^{2}<<1$, the results of calculations for the module and the impedance phase practically coincide with the numerical solution to equation (1). Therefore, when calculating the impedance to $20 \mathrm{MHz}$ we use formula (1).

\section{Conclusion}

Two-layer media is considered the "ultimate dielectric layer thickness" - conductive unlimited depth into medium. This two-layer media over a wide frequency range radio waves allowed to allocate a small amount and get analytical expression (1) for the surface impedance. This expression for the impedance compared with numerical calculation for multi-layered media, which showed a satisfactory agreement.

Acknowledgments. This article is prepared on the budget draft of Laboratory Electromagnetic Diagnostics Institute of Physical Materials Science of the Siberian Branch RAS.

\section{References}

1. Balkhanov V. K. and Bashkuev Yu. B., Principles of the Method of Surface Impedance (Publishing House of the Buryat Scientific Center SB RAS, Ulan-Ude, 2005).

2. Makarov G.I., Novikov V.V. and Rybachek S.T., Propagation of Electromagnetic Waves over the Earth Surface (Nauka, Moscow, 1991).

3. James R. Wait. Electromagnetic waves in stratified media. (Oxford, New York, Pergamon Press, 1996).

4. Bashkuev Yu. B., Khaptanov V. B. and Dembelov M. G., Tech. Phys. Lett. 36, 136 (2010).

5. Balkhanov V.K. and Bashkuev Yu.B., Technical Physics, 2017, Vol. 62, No 4, pp. 619-624. DOI: 10.1134/S106378421704003X

6. Angarkhaeva L.Kh. The software package "Impedance" to meet the challenges of the radioimpedans sensing: the certificate of official registration programs. Russian Federation / Statement 11.04.2002; Sign up 06.06.2002.

7. Bashkuev Yu.B., Naguslaeva I.B., Khaptanov V.B. and M. G. Dembelov M.G. Technical Physics, 2016, Vol. 61, No. 2.

8. Balkhanov V.K., Bashkuev Yu.B. Angarkhaeva L.Kh. and other, Technical Physics, 2014, Vol. 59, No. 11, pp. 1679-1683. DOI: 10.1134/S106378421411005X. 